\title{
The contact theorem and sum rules in the theory of electrical double layer
}

\author{
M.F.Holovko ${ }^{1}$, J.P.Badiali ${ }^{2}$ \\ 1 Institute for Condensed Matter Physics \\ of the National Academy of Sciences of Ukraine, \\ 1 Svientsitskii Str., 79011 Lviv, Ukraine \\ 2 laboratoire de Electrochimie \\ et Chimie Analytique ENSCP-UPMC, \\ 11 rue P. et M. Curie, 75231 Cedex 05,Paris,France
}

Received April 19, 2005

The specific sum rules in the theory of electrical double layer are formulated as the consequence of contact theorem. They concern the correlation functions of electrolyte when one ion is located on the surface. A simple interpretation of the obtained relations is given.

Key words: electrolyte solution, double layer, contact theorem, sum rules

PACS: 05.20.Jj, 05.30.Fk, 05.10.Zn

It is a great pleasure for us to contribute this paper to the special issue dedicated to the $70^{\text {th }}$ anniversary of D. Henderson, one of the true leaders in the liquid state theory. We have had a pleasure and benefit of collaborating with him during long time.

The contact theorem is one of the few exact results in the theory of electrical double layer $[1,2]$. It is based on force balance consideration between the bulk pressure and the force exerted by the fluid particles on the container wall. A formal derivation of the contact theorem can be obtained by the direct integration of the Yvon-Born-Green (YBG) equation [3]. For a restricted primitive model (RPM) electrolyte at a uniformly charged flat hard wall, the contact theorem reduces to the relation $[1-3]$

$$
k T \rho\left(z=\frac{R}{2}\right)=P+\frac{\varepsilon E^{2}}{8 \pi},
$$


where $\rho(z=R / 2)$ is the contact value of the density profile $\rho(z)=\rho_{+}(z)+\rho_{-}(z), R$ is the diameter of ions, $k$ is the Boltzmann constant, $T$ is the absolute temperature, $P$ is the bulk pressure of the electrolyte, $\varepsilon$ is the solvent dielectric permittivity, $E$ is the electric field at contact related to the uniform surface charge of the wall $\tau=\varepsilon E / 4 \pi$.

The first term in relation (1) is connected with the contact theorem for noncharged wall

$$
k T \rho\left(z=\frac{R}{2}, E=0\right)=P .
$$

The second term describes the effect of charged wall

$$
k T \Delta \rho\left(z=\frac{R}{2}, E\right)=\frac{\varepsilon E^{2}}{8 \pi},
$$

where

$$
\rho(z)=\rho(z, E=0)+\Delta \rho(z, E) .
$$

As the consequence of the contact theorem

$$
\begin{aligned}
k T \frac{\partial \rho(z=R / 2)}{\partial E} & =\frac{\varepsilon E}{4 \pi}, \\
k T \frac{\partial^{2} \rho(z=R / 2)}{\partial E^{2}} & =\frac{\varepsilon}{4 \pi}, \\
\frac{\partial^{n} \rho(z=R / 2)}{\partial E^{n}} & =0, \quad \text { for } n \geqslant 3 .
\end{aligned}
$$

We can consider the Gouy-Chapman (GC) theory [4,5] as a simple example of the theory within the framework of which the relation (3) is satisfied.

In GC approximation

$$
\rho\left(z=\frac{R}{2}\right)=\rho \operatorname{ch}\left(\beta e \Psi\left(z=\frac{R}{2}\right)\right),
$$

where $\beta=1 /(k T), \rho$ is bulk density of ions, $e$ is elementary electrical charge, $\Psi(z=R / 2)$ is electrostatic potential drop at the wall which satisfies the equation

$$
\operatorname{sh}\left(\frac{\beta}{2} e \Psi\left(z=\frac{R}{2}\right)\right)=\frac{1}{2 \kappa} \beta E e,
$$

$\kappa=\left(4 \pi \beta e^{2} \varepsilon^{-1} \rho\right)^{1 / 2}$ is the Debye-Huckel parameter.

For the first derivative we have

$$
\frac{\partial \rho(z=R / 2)}{\partial E}=\frac{2 \rho \beta e}{\kappa} \operatorname{sh}\left(\frac{1}{2} \beta e \Psi\left(z=\frac{R}{2}\right)\right)=\frac{\beta \varepsilon E}{4 \pi}
$$

in accord with equation (5). For the second and for the higher derivatives we have relations (6) and (7), respectively. 
In general the derivative $\left[\partial^{n} \rho(z=R / 2)\right] / \partial E^{n}$ can be treated as n-th response of the electrolyte to the change of surface charge. This response can be expressed explicitly in terms of correlation functions of $(n+1)$ ions, one of which is fixed on the wall. As a result, the relations (5)-(7) lead to the new special exact relations between correlation functions of electrolyte near the wall. The analysis of such relations is the subject of this paper.

The first, second and higher derivatives are connected with two, three and higher structure functions

$$
\begin{aligned}
S_{\alpha \beta}(12)= & \delta_{\alpha \beta} \delta(12) \rho_{\beta}(2)+\rho_{\alpha}(1) \rho_{\beta}(2) h_{\alpha \beta}(12) \\
S_{\alpha \beta \gamma}(123)= & \delta_{\alpha \beta} \delta_{\beta \gamma} \delta(12) \delta(23) \rho_{\gamma}(3) \\
& +\delta_{\alpha \gamma} \delta(13) \rho_{\beta}(2) \rho_{\gamma}(3) h_{\beta \gamma}(23)+\delta_{\alpha \beta} \delta(12) \rho_{\alpha}(1) \rho_{\gamma}(3) h_{\alpha \gamma}(13) \\
& +\delta_{\beta \gamma} \delta(23) \rho_{\alpha}(1) \rho_{\beta}(2) h_{\alpha \beta}(12) \\
& +\rho_{\alpha}(1) \rho_{\beta}(2) \rho_{\gamma}(3) h_{\alpha \beta \gamma}(123), \quad \ldots
\end{aligned}
$$

by the relations

$$
\begin{aligned}
\frac{\partial \rho(1)}{\partial E}= & -\beta \sum_{\alpha, \beta} \int \mathrm{d} 2 \frac{\partial W_{\beta}(2)}{\partial E} S_{\alpha \beta}(12), \\
\frac{\partial^{2} \rho(1)}{\partial E^{2}}= & \beta^{2} \sum_{\alpha, \beta, \gamma} \int \mathrm{d} 2 \int \mathrm{d} 3 \frac{\partial W_{\beta}(2)}{\partial E} \frac{\partial W_{\beta}(3)}{\partial E} S_{\alpha \beta \gamma}(123), \\
\vdots & \\
\frac{\partial^{n} \rho(1)}{\partial E^{n}}= & (-1)^{n} \beta^{n} \sum_{\alpha_{1} \ldots \alpha_{n+1}} \int \mathrm{d} 2 \cdots \int \mathrm{d}(n+1) \frac{\partial W_{\alpha_{2}}(2)}{\partial E} \cdots \frac{\partial W_{\alpha_{n+1}}(n+1)}{\partial E} \\
& \times S_{\alpha_{1} \cdots \alpha_{n+1}}(12 \cdots n+1),
\end{aligned}
$$

where

$$
W_{\alpha}(z)= \begin{cases}\infty, & z<R / 2 \\ -z_{\alpha} e E z \varepsilon^{-1}, & z>R / 2\end{cases}
$$

the potential of interaction between surface and ions of species $\alpha, z_{+}=-z_{-}=1$; $1,2, \ldots$ denote the coordinates of corresponding ions, $\int \mathrm{d} 2=\int \mathrm{d} \overline{r_{2}}, \delta_{\alpha \beta}$ - is the Kroneker symbol, $\delta(12)$ is the corresponding $\delta$-function; $h_{\alpha \beta}(12), h_{\alpha \beta \gamma}(123) \ldots$ are pair, triplet and higher correlation functions of ions.

Since $\partial W_{\alpha}(z) / \partial E=-z_{\alpha} e z \varepsilon^{-1}$, the comparison (12)-(14) and (5)-(7) and the application of local electroneutrality condition $[6]$

$$
e z_{\alpha}+\sum_{\beta} e z_{\beta} \int \mathrm{d} 2 \rho_{\beta}(2) h_{\alpha \beta}(12)=0
$$


leads to the following identities

$$
\begin{gathered}
\frac{\partial \rho(z=R / 2)}{\partial E}=\frac{e \beta}{\varepsilon} \sum_{\alpha \beta} z_{\beta} \rho_{\alpha}\left(z_{1}=\frac{R}{2}\right) \int \mathrm{d} \bar{r}_{2}\left(z_{2}-\frac{R}{2}\right) \\
\times \rho_{\beta}\left(z_{2}\right) h_{\alpha \beta}\left(z_{1}=\frac{R}{2}, \bar{r}_{12}, z_{2}\right)=\beta \frac{\varepsilon E}{4 \pi}, \\
\frac{\partial^{2} \rho(z=R / 2)}{\partial E^{2}}=\beta \frac{\varepsilon}{4 \pi}=\frac{e^{2} \beta^{2}}{\varepsilon^{2}}\left[\frac{1}{2} R \sum_{\alpha \beta} z_{\alpha} z_{\beta} \rho_{\alpha}\left(z_{1}=\frac{R}{2}\right)\right. \\
\times \int \mathrm{d} \bar{r}_{2}\left(2 z_{2}-\frac{R}{2}\right) \rho_{\beta}\left(z_{2}\right) h_{\alpha \beta}\left(z_{1}=\frac{R}{2}, \bar{r}_{12}, z_{2}\right)+\sum_{\alpha \beta} z_{\beta}^{2} \rho_{\alpha}\left(z_{1}=\frac{R}{2}\right) \\
\times \int \mathrm{d} \bar{r}_{2} z_{2}^{2} \rho_{\beta}\left(z_{2}\right) h_{\alpha \beta}\left(z_{1}=\frac{R}{2}, \bar{r}_{12}, z_{2}\right)+\sum_{\alpha \beta \gamma} z_{\beta} z_{\gamma} \rho_{\alpha}\left(z_{1}=\frac{R}{2}\right) \\
\left.\quad \times \int \mathrm{d} \bar{r}_{2} \int \mathrm{d} \bar{r}_{3} z_{2} z_{3} \rho_{\beta}\left(z_{2}\right) \rho_{\gamma}\left(z_{3}\right) h_{\alpha \beta \gamma}\left(z_{1}=\frac{R}{2}, z_{2}, z_{3}, \bar{r}_{12}, \bar{r}_{23}\right)\right] .
\end{gathered}
$$

For $n \geqslant 3$

$$
\begin{aligned}
\frac{\partial^{n} \rho(z=R / 2)}{\partial E^{n}}= & \left(\frac{e \beta}{\varepsilon}\right)^{n} \sum_{\alpha_{1} \cdots \alpha_{n+1}} z_{\alpha_{2}} \cdots z_{\alpha_{n+1}} \int \mathrm{d} 2 \cdots \int \mathrm{d}(n+1) z_{2} \cdots z_{n+1} \\
& \times S_{\alpha_{1}, \alpha_{2}, \ldots, \alpha_{n+1}}\left(z_{1}=\frac{R}{2}, 23 \cdots n+1\right)=0 .
\end{aligned}
$$

The identities (16)-(18) are the main result of this paper. They follow directly from the contact theorem and can be considered as specific sum rules for the correlation functions electrolyte near the charged hard wall. The first of them was formulated by Blum, Henderson et al [7] and has the following meaning.

$$
e \sum_{\beta} z_{\beta} \rho_{\alpha}\left(z_{1}=\frac{R}{2}\right) h_{\alpha \beta}\left(z_{1}=R / 2, \bar{r}_{12}, z_{2}\right) \rho_{\beta}\left(z_{2}\right)
$$

is the excess charge density relatively to the ion with the charge $e z_{\alpha}$ located at the surface at $z_{1}=R / 2$.

$$
e \sum_{\beta} z_{\beta} \rho_{\alpha}\left(z_{1}=\frac{R}{2}\right) \int \mathrm{d} \bar{r}_{2}\left(z_{2}-\frac{R}{2}\right) h_{\alpha \beta}\left(z_{1}=R / 2, \bar{r}_{12}, z_{2}\right) \rho_{\beta}\left(z_{2}\right)
$$

can be considered as dipolar polarization of electrolyte related to the ion of species $\alpha$ located at $z_{1}=R / 2$. Thus, the relation (16) means that the sum of dipolar polarizations relatively to cation and anion located at the wall is equal to the surface charge $\tau=(\varepsilon E) /(4 \pi)$. For the noncharged surface both polarizations relatively to 
cation and anion have the same values but the opposite signs. As a result their sum is equal zero.

The relation (17) includes the quadratic response of electrolyte

$$
e^{2} \sum_{\beta \gamma} z_{\beta} z_{\gamma} S_{\alpha \beta \gamma}\left(z_{1}=\frac{R}{2}, 2,3\right),
$$

when the ion of species $\alpha$ is located on the wall and

$$
e^{2} \sum_{\beta \gamma} z_{\beta} z_{\gamma} \int \mathrm{d} 2 \mathrm{~d} 3 z_{2} z_{3} S_{\alpha \beta \gamma}\left(z_{1}=\frac{R}{2}, 2,3\right)
$$

can be considered as quadrupolar polarization of electrolyte related to the ion of species $\alpha$ located at $z_{1}=R / 2$. According to (17) the sum of the quadrupolar polarizations relatively to the cation and anion located at the wall is constantly equal $\varepsilon / 4 \pi$ which does not depend on the wall charge. Such conclusion is the result of the semi-infinity symmetry of electrical double layer.

Due to semi-infinity symmetry of electrical double layer the sum of octupolar and higher polarizations of electrolyte relatively to cation and anion located at the wall is equal to zero. This is the meaning of the relations (18).

\section{Acknowledgement}

The collaboration of our laboratories takes place in the framework of NASUCNRS agreement within the project No. 126111. We thank D. di Caprio for the useful discussion.

\section{References}

1. Henderson D., Blum L., Lebowitz J.L., J. Electroanal. Chem., 1979, 102, 315.

2. Henderson D., Blum L., J. Chem. Phys., 1981, 75, 2025.

3. Carnie S.L., Chan D.Y.C, J. Chem. Phys., 1981, 74, 1293.

4. Gouy G., J. Phys., 1910, 9, 457; Chapman D.L., Phil. Mag., 1913, 25, 475.

5. Henderson D., Blum L., J. Chem. Phys., 1978, 69, 544.

6. Carnie S.L., Chan D.Y.C, J. Chem. Phys. Lett., 1981, 77, 437.

7. Blum L., Henderson D., Lebowitz J.L., Gruber Ch., Martin Ph.A., J. Chem. Phys., 1981, 75, 5974. 


\title{
Контактна теорема і правила сум у теорії подвійного електричного шару
}

\author{
М.Ф.Головко ${ }^{1}$, Ж.-П.Бадіалі ${ }^{2}$
}

1 Інститут фізики конденсованих систем НАН України, 79011 Львів, вул. Свєнціцького, 1

2 Лабораторія електрохімії та аналітичної хімії, Вища національна школа хімії в Парижі, Університет П'єра і Марі Кюрі, Франція, Париж, вул.П'єра і Марі Кюрі,11

Отримано 19 квітня 2005 р.

На основі контактної теореми сформульовані специфічні правила сум в теорії подвійного електричного шару. Вони стосуються кореляційних функцій електроліту, коли один з іонів знаходиться на поверхні. Дана проста інтерпретація отриманих співвідношень.

Ключові слова: розчин електроліту, подвійний шар, контактна теорема, правила сум

PACS: 05.20.Jj, 05.30.Fk, 05.10.Zn 